\title{
Cognitive Motion Extrapolation and Cognitive Clocking in Prediction Motion Tasks
}

\author{
Patricia R. DeLucia and Gregory W. Liddell \\ Texas Tech University
}

\begin{abstract}
An interruption paradigm was used to measure judgments that rely on cognitive extrapolation of approach and lateral motion. In some conditions the pattern of errors was consistent with that obtained with time-to-contact (TTC) judgments measured with a prediction motion (PM) task. Also, the slope of the relationship between estimated and actual TTC in judgments of approaching objects decreased when visual information about the environment between the observer and the display was minimized. Moreover, the accuracy of relative duration judgments of visual (but not auditory) stimuli decreased when a PM task was performed concurrently. Results are consistent with the notion that PM tasks involve cognitive motion extrapolation rather than solely a clocking process that counts down TTC.
\end{abstract}

Information in the optic array specifies when an object will hit or pass the observation point and when it will hit another designated object (e.g., Bootsma \& Oudejans, 1993; Lee, 1974; Tresilian, 1990, 1991). Results suggest that observers can use or are sensitive to such optical information about time to contact (TTC; e.g., tau, Lee, 1974) ${ }^{1}$ in various tasks (e.g., Bootsma \& Oudejans, 1993; Kaiser \& Mowafy, 1993; Schiff \& Detwiler, 1979; Schiff \& Oldak, 1990; Todd, 1981). However, results also suggest that judgments about TTC are constrained by scene and threshold factors and can be based on multiple sources of information (e.g., DeLucia, 1991, 1995; DeLucia \& Warren, 1994; Schiff \& Detwiler, 1979; Schiff \& Oldak, 1990; Tresilian, 1995). Moreover, the finding that observers' judgments about TTC are consistent with their use of optical TTC does not preclude the possibility that such judgments are constrained by cognitive operations, especially in complex situations when several objects are judged (DeLucia \& Novak, 1997; Novak, 1998) or when objects are not continuously in view.

Patricia R. DeLucia and Gregory W. Liddell, Department of Psychology, Texas Tech University.

This research was supported by the Research Enhancement Fund at Texas Tech University. Portions of this research were presented at the November 1994 annual meeting of the Psychonomic Society, St. Louis, Missouri.

We are grateful to Mary $\mathrm{K}$. Kaiser for comments that led to the inclusion of Experiment 3, to Joseph S. Lappin for direction in the computations of the Weber fractions, to James $R$. Tresilian and the late Jason $\mathrm{E}$. Albrecht for helpful suggestions on earlier drafts, and to Gregory H. Mumma for suggestions about trend analyses. We also thank Jeff Maresh and Robert Todd of Engineering Solutions, Inc., for technical assistance.

Correspondence concerning this article should be addressed to Patricia R. DeLucia, Department of Psychology, Mail Stop \#2051, Texas Tech University, Lubbock, Texas 79409-2051. Electronic mail may be sent to djxpd@ttacs.ttu.edu.

\section{Cognitive Operations in Judgments About TTC}

Tresilian (1995) argued that task variables affect whether performance involves cognitive information processing and whether optical TTC is used. He proposed that prediction motion (PM) tasks may involve cognitive operations. In PM tasks, an object moves toward a target and disappears; observers respond at the time that they think the object would reach the target. In previous studies (e.g., Schiff \& Detwiler, 1979; Schiff \& Oldak, 1990), estimates of TTC increased as actual TTC increased, which suggested that judgments were based on optical TTC. However, observers estimated TTC inaccurately when actual TTC exceeded 2-3 s, and the inaccuracy increased as actual TTC increased.

One of the features of PM tasks that implicates cognitive operations is that the response occurs in the absence of visual information (Jagacinski, Johnson, \& Miller, 1983; Schiff \& Oldak, 1990; Tresilian, 1995). We consider two classes of cognitive operations that may be involved in PM tasks.

The first class, cognitive motion extrapolation (CME), involves an internal or cognitive representation of the object's visible motion. For example, observers may develop a cognitive model of the object's visible motion and use this model to extrapolate the object's motion after it disappears and to estimate TTC (Schiff \& Oldak, 1990; Tresilian, 1995). The observed errors in TTC judgments can be attributed to an inaccurate internal model of the object's motion (Jagacinski et al., 1983). Schiff and Oldak (1990) suggested that information about TTC is available in any part of an object's motion; thus, whether the spatiotemporal gap being judged transforms the task into a cognitive one depends on the theoretical perspective that one espouses. From an ecological orientation (e.g., Gibson, 1979), the

'For brevity, the term time to contact is used generically to denote the time remaining before an object contacts the observation point or a target or passes it without collision. 
termination of sensory input from a motion event is not relevant to its perceptual character (Schiff \& Oldak, 1990); thus, CME is unnecessary. However, such reasoning does not explain how observers time their response to coincide with their judgment of TTC when optical TTC is not available at the time of response initiation (see Tresilian, 1995).

Imagery, eye movements, and attentional shifts may accompany CME. For example, observers may imagine that the object continues to move after it disappears and may respond when the imagined object reaches the target; observers may track the object's visible motion with eye movements and, after the object disappears, continue the eye movements until the end point is reached; or tracking may involve an attentional "spotlight" (Lyon \& Waag, 1995). We assume that a cognitive representation of the object's motion is involved in all of these cases.

Rosenbaum (1975) proposed that observers may directly extend the object's motion after it disappears, possibly relying on a form of imagery. His informal observations of eye movements during the object's hidden motion in a PM task indicated that observers most often moved their eyes continuously as if the target was still visible and sometimes moved their eyes in regular intervals in the marker's direction. However, differences in eye movements were not consistent across experimental conditions or observers. Also, Peterken, Brown, and Bowman (1991) reported that position prediction was equivalent when eye tracking was encouraged and when it was prevented via instructions to track the object or fixate a static line, and they refuted the hypothesis that visual tracking or eye movements are necessary for successful predictions. It would be difficult to determine whether the presence of eye movements indicates that eye movement information per se is used to extrapolate motion or whether eye movements are simply a by-product of visual imagery or an attentional spotlight (see also Lyon \& Waag, 1995).

A second class of cognitive operations that may be involved in PM tasks involves a clocking, or timing, mechanism. For example, Tresilian (1995) proposed a cognitive process that involves a clock process. Specifically, observers may initially estimate TTC before the object's disappearance and may use a clocking process to count down time. The response is initiated when the time reaches the estimated TTC (minus the time for the button response). Such a process can potentially explain how observers time a response to coincide with their judgment of TTC after an object disappears. In subsequent discussions of such a clocking process, we assume that the initial estimate of TTC is based on optical TTC.

PM tasks may involve a clocking process that does not rely on optical TTC. For example, to use CME, a temporal process would seem necessary to update the object's extrapolated position (and corresponding imagery) at each point in time until it reaches the end point. Alternatively, observers may compute the object's hidden time (TTC) from the ratio of exposed distance to hidden distance and the duration of the object's visible motion; the clock would count the latter duration and the hidden time (Rosenbaum, 1975; see also Lyon \& Waag, 1995).

Lyon and Waag (1995) classified hypotheses about the mechanisms that underlie the ability to extrapolate motion as either tracking hypotheses or timing hypotheses. Timing hypotheses involve a central timing mechanism that clocks the object's visible motion; this elapsed time is the basis for how long the observer waits before responding. Tracking hypotheses involve mechanisms that follow the target's motion after it disappears. We include tracking in the more general class of processes that rely on a cognitive representation of the object's motion.

We hypothesize that observers use CME accompanied by imagery. We propose that they internalize the object's visible motion in some manner, for example, with a cognitive model or memory representation that is used to extrapolate the object's motion after it disappears and to update the corresponding imagery of the motion. The use of imagery is consistent with earlier notions about PM tasks (Kaiser \& Mowafy, 1993; Rosenbaum, 1975; see also Schiff \& Oldak, 1990, p. 310) and with subjective reports from participants in our experiments. Furthermore, it seems that whether observers use CME or solely a cognitive clock that counts down TTC to perform a PM task, it would be difficult to rule out some type of temporal process. Therefore, the objective of the research described here was to evaluate whether the PM task would involve CME rather than solely a cognitive clock that relies on optical TTC.

\section{Experiment 1}

The PM task, as used in previous studies to measure judgments about TTC, does not facilitate an empirical evaluation of whether observers rely on CME rather than, or in addition to, optical TTC. The increase in the magnitude of response error with larger TTC values can be attributed to inaccuracies in the use of optical TTC or in cognitive operations that occur after the object disappears (and that decay with time; Rosenbaum, 1975). In other words, the PM task does not permit a direct test of whether observers cognitively extrapolate motion. Therefore, we sought an indirect method that would facilitate an evaluation of whether observers use CME to aid their judgments in the PM task. We wanted a task that would require observers to use CME rather than optical TTC so that we could compare errors in such a task with errors in the PM task.

Specifically, we used an interruption paradigm (IP; Cooper, 1989; Cooper, Gibson, Mowafy, \& Tataryn, 1987; see also Larish \& Andersen, 1995). In the IP, an object moves at a constant rate and then disappears for a variable duration. It then reappears either at the correct position in its trajectory (assuming a constant velocity during the blackout) or at a position that is more advanced (i.e., overshoot) or less advanced (i.e., undershoot) than the correct position. The observers report whether the object reappears at the correct position in its transformational trajectory. Cooper (1989) characterized this method as one in which observers "are asked to extrapolate internally a continuous external trans- 
formation of an object that is momentarily interrupted" (p. 109).

In the IP, after the object disappears, observers are not told where or when it will reappear. A strategy in which time is counted down from an initially estimated TTC is not viable in this task; TTC is not relevant. Thus, we argue that in the IP observers cognitively extrapolate motion rather than rely on optical TTC. If observers use CME in the PM and IP tasks, the pattern of response errors should be similar. For example, underestimations of TTC in the PM task imply that at a given point in time after the object disappears, observers perceive the object as being farther along in its path than it actually is. This kind of error is measured directly with the IP; if the same object reappears in a position that is more advanced than the correct one, observers would judge the reappearance position as correct. Furthermore, one can measure how the accuracy of CME varies with time by manipulating the blackout duration in the IP, which is analogous to TTC in the PM task. The larger errors that occur with relatively large TTCs (i.e., long blackout durations) imply that the accuracy of extrapolation decreases over time (Rosenbaum, 1975; see also Larish \& Andersen, 1995). In the IP, the effects of blackout duration on the accuracy of CME can be measured independently of TTC.

In Experiment 1, we measured judgments of approach and lateral motion with the IP to determine whether the pattern of responses would be similar to that in previous studies of TTC (Schiff \& Detwiler, 1979; Schiff \& Oldak, 1990). In the latter, the accuracy of TTC judgments in a PM task decreased as actual TTC increased, and errors typically consisted of underestimations; furthermore, TTC judgments were more accurate with lateral motion than with approach motion. Thus, if performance in the PM task reflects CME, judgments in the IP should be more accurate with short blackout durations, should be biased toward overshoots (analogous to underestimations of TTC in a PM task) with approach motion, and should be more accurate with lateral motion. Such results would suggest that a cognitive clock based on TTC is not necessary to obtain a pattern of extrapolation errors that characterizes performance in a PM task.

\section{Method}

Participants. Sixteen students at Texas Tech University received credit toward an introductory psychology course, had normal or corrected visual acuity, and were not informed of the experimental hypotheses.

Displays. Computer simulations were created on an MS-DOS $486 / 50 \mathrm{MHz}$ computer with $640 \times 350$ pixel resolution and a presentation rate of 23.4 frames/s on a $35.56-\mathrm{cm}$ (14-in.) monitor. Displays consisted of perspective white-on-black drawings of 3-D scenes in which an object underwent approach or lateral motion. Schematic representations of the scenes are shown in Figure 1. The optical parameters of the moving object are shown in Tables 1 and 2.

At the beginning of each scene, the object was stationary for $1 \mathrm{~s}$; it then moved at a constant speed for $2 \mathrm{~s}$. In lateral motion scenes, the object moved rightward toward a vertical "finish line" while located on a "road." In approach scenes, an object moved along the depth axis toward a finish line (two poles) while located on a ground plane. We designed the scenes to be similar to those
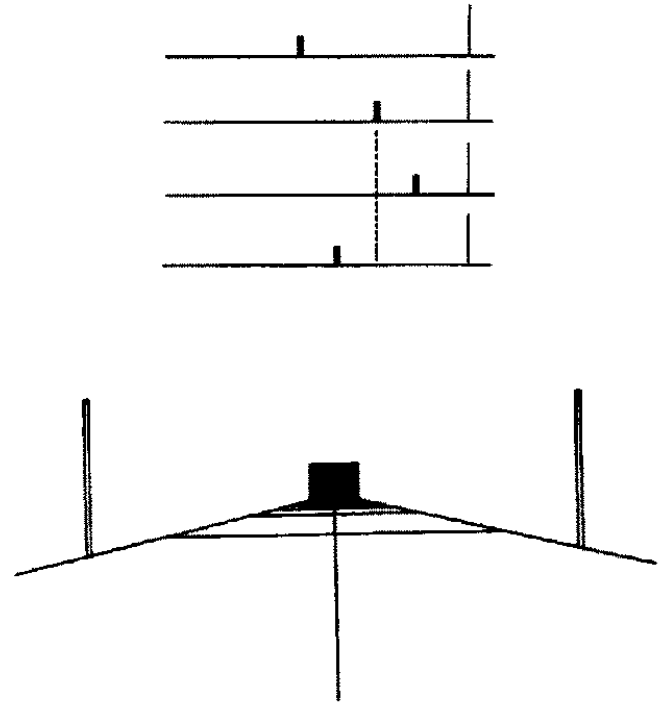

Figure 1. Top drawing: schematic representation of lateral motion scenes in Experiment 1 (top line shows position before disappearance; second line from top shows correct reappearance position; third line from top shows an overshoot; bottom line shows an undershoot). Bottom drawing: schematic representation of approach scenes.

used in previous TTC studies (Schiff \& Detwiler, 1979) and to accommodate the IP.

The object disappeared when it was at either a near or a far distance from the finish line. Objects that disappeared at a far distance started farther and moved faster in virtual space than objects that disappeared at a near distance. We refer to this manipulation as the "final distance" to be consistent with earlier TTC studies (e.g., Schiff \& Detwiler, 1979). With lateral motion scenes in which the object disappeared at a near distance, the object moved through $1.44^{\circ}$ of visual angle; in far-distance scenes, the corresponding value was $2.88^{\circ}$. With approach motion, the object's optical expansion was $0.95^{\circ}$ and $0.73^{\circ}$ in near- and far-distance scenes, respectively.

After the object moved, it disappeared for either 1.5 or $3.2 \mathrm{~s}$ and then reappeared while moving. On half the trials, the object reappeared at the correct position in its trajectory, assuming a constant velocity during the blackout. On the other trials, the object reappeared at a position that was less advanced than the correct position (undershoots) or more advanced than the correct position (overshoots; see Figure 1).

When we manipulated the object's reappearance position, we defined the deviation between the correct reappearance position

Table 1

Experiment 1: Degrees of Visual Angle Subtended by the Gap Between the Object and the Finish Line (Lateral) or by the Height of the Moving Object (Approach) on the First Frame and the Last Frame (Before Disappearance)

\begin{tabular}{|c|c|c|c|c|}
\hline \multirow{2}{*}{$\begin{array}{l}\text { Final } \\
\text { distance }\end{array}$} & \multicolumn{2}{|c|}{ Lateral } & \multicolumn{2}{|c|}{ Approach } \\
\hline & First frame & Last frame & First frame & Last frame \\
\hline $\begin{array}{l}\text { Near } \\
\text { Far }\end{array}$ & $\begin{array}{r}9.489 \\
13.246\end{array}$ & $\begin{array}{r}8.045 \\
10.369\end{array}$ & $\begin{array}{l}2.742 \\
1.647\end{array}$ & $\begin{array}{l}3.687 \\
2.380\end{array}$ \\
\hline
\end{tabular}


Table 2

Experiment 1: Optical Parameters of Correct-Reappearance Scenes

\begin{tabular}{ccc}
\hline & \multicolumn{2}{c}{ Blackout duration } \\
\cline { 2 - 3 } Final distance & $1.5 \mathrm{~s}$ & $3.2 \mathrm{~s}$ \\
\hline & Lateral motion \\
Near & 1.11 & \\
Far & 2.23 & 2.35 \\
& Approach motion \\
Near & 5.01 & \\
Far & 3.61 & 8.32 \\
\hline
\end{tabular}

Degrees of visual angle subtended by the distance between disappearance position and reappearance position. bDegrees of visual angle subtended by the object's height upon reappearance.

and the incorrect reappearance positions in terms of the optical change that was characteristic of each motion. Specifically, for lateral motion, the object reappeared at a position that was displaced laterally from the correct position; the displacement was $0.5^{\circ}, 1^{\circ}$, or $2^{\circ}$ more advanced or less advanced than correct. For approach motion, the object reappeared at a position that was displaced along the depth axis from the correct position; the displacement resulted in an optical size that was $0.5^{\circ}, 1^{\circ}$, or $2^{\circ}$ larger or smaller than that produced with the correct reappearance position (see Table 2 for additional optical parameters of the scenes). This method enhanced the comparison of the PM and IP tasks; in PM tasks, an object's rate of optical expansion (in approach motion) and rate of optical gap constriction (in lateral motion) are essential components of the information that specifies TTC (see Bootsma \& Oudejans, 1993; Tresilian, 1990, 1991).

Design and procedure. Observers viewed the displays biocularly from $0.61 \mathrm{~m}$, and head movements were minimized with a chin rest. The observers were instructed to indicate, using mouse buttons, whether the object reappeared at the correct position in its trajectory assuming a constant velocity during the blackout.

To strengthen the IP as a measure of CME, we instructed observers to visualize or imagine the motion that would have occurred had the object remained visible. ${ }^{2}$ Feedback was not provided, and the percentage of trials in which observers reported that the object reappeared in the correct position was measured. Trials in which the observer responded before the object reappeared were omitted. Every observer viewed 288 trials that included all levels of blackout duration, reappearance position, and final distance presented in a random order. Motion trajectory was a between-subjects variable and was balanced for gender. Fourteen practice trials were provided but were not analyzed.

\section{Results and Discussion}

We evaluated whether responses were biased toward undershoots, overshoots, or correct reappearance positions. Bias toward overshoots is consistent with the notion that a property of CME leads observers to perceive that, at a given point in time after the object disappears, the object is farther along in its path than it actually is; that is, the mental process of extrapolation "speeds up" during the blackout (Cooper, 1989). This same property of CME would lead observers to underestimate TTC in a PM task. Similarly, bias toward undershoots is consistent with overestimations of TTC in a
PM task. To identify such biases, we created response curves by plotting the mean percentage of trials in which observers reported that the object reappeared in the correct position (the percentage reported "correct") as a function of the deviation between the actual reappearance position and the objectively correct reappearance position (Cooper, 1989).

We analyzed the data in several ways: First, for each level of motion and blackout duration, we observed where the peak of the response curve occurred; that is, we identified the reappearance position that was most often reported to be correct (Cooper, 1989). (Note that relatively flatter curves indicated poorer discrimination among the reappearance positions.) To evaluate more quantitatively whether response curves contained a peak or were flat, we used trend analyses. Second, we visually inspected the symmetry of the curves to evaluate response biases toward undershoots or overshoots (Cooper, 1989). To evaluate response biases more quantitatively, we averaged the percentage reported to be correct across the three levels of undershoots and compared the result with the average of the three levels of overshoots (with an analysis of variance [ANOVA]). Third, we used an ANOVA to evaluate the effects of the independent variables on performance. ${ }^{3}$

Lateral motion. As shown in Figure 2 (top left panel), the objectively correct reappearance position (a deviation of zero) was chosen most often as correct when the blackout duration was $1.5 \mathrm{~s}$ or $3.2 \mathrm{~s}$. The quadratic component was significant and accounted for the most variance: $1.5 \mathrm{~s}$, linear $F(1,47)=5.92, p<.025, R^{2}=10.90 \%$, quadratic $F(1,46)=46.88, p<.001, R^{2}=43.69 \% ; 3.2 \mathrm{~s}$, quadratic $F(1,46)=27.11, p<.001, R^{2}=30.95 \%$. Although visual inspection of the 1.5-s curve suggested an asymmetry and possible bias toward overshoots, the mean percentage reported to be correct for overshoots was not significantly different from that for undershoots with either blackout duration. The results (a) indicate that responses were fairly symmetrical around the correct reappearance position and (b) are consistent with the relatively accurate judgments of lateral motion (compared with approach motion) obtained in previous TTC studies (Schiff \& Oldak, 1990).

Results of a $2 \times 2 \times 7$ repeated measures ANOVA (Final Distance $\times$ Blackout Duration $\times$ Reappearance Position) indicated that reappearance position accounted for the most variance, $F(6,42)=10.56, p<.0018, M S E=1,439.54$, $\omega^{2}=30.13 \%$. There was also a three-way interaction among reappearance position, blackout duration, and final distance, $F(6,42)=3.34, p<.041, M S E=336.40, \omega^{2}=1.72 \%$.

Approach motion. As shown in Figure 2 (top left panel), a $0.5^{\circ}$ overshoot was chosen most often as correct when the blackout duration was $1.5 \mathrm{~s}$. The linear, quadratic, and cubic components were significant: linear, $F(1,47)=67.29, p<$

\footnotetext{
${ }^{2}$ A postsession questionnaire indicated that all observers reported that they mentally extrapolated or imagined the object's motion when it disappeared.

${ }^{3}$ We obtained the same results with analyses of percentage reported to be correct as with analyses of the arcsine transformation of this percentage. The probability values from the results of the analyses of variance reflect Greenhouse-Geisser corrections.
} 


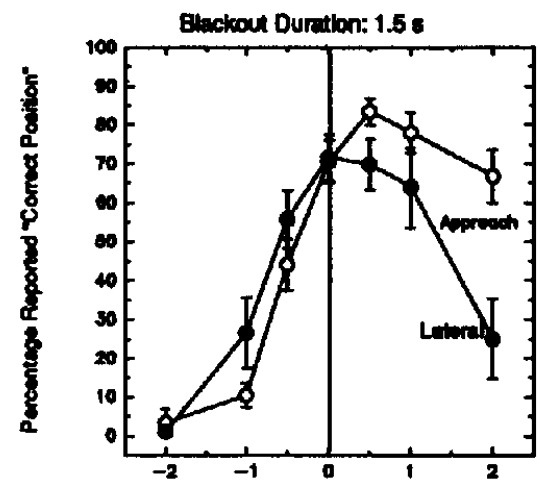

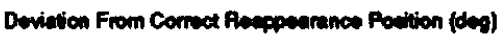

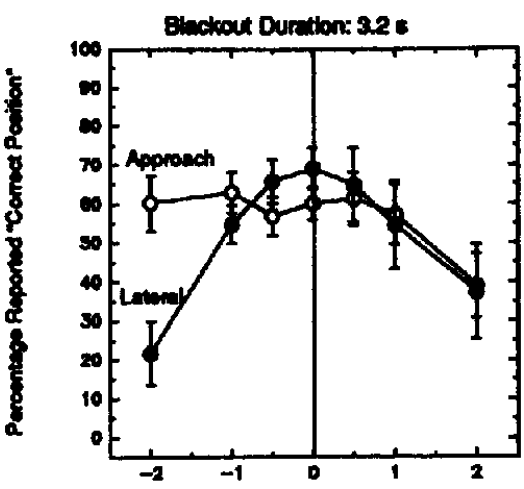

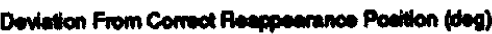

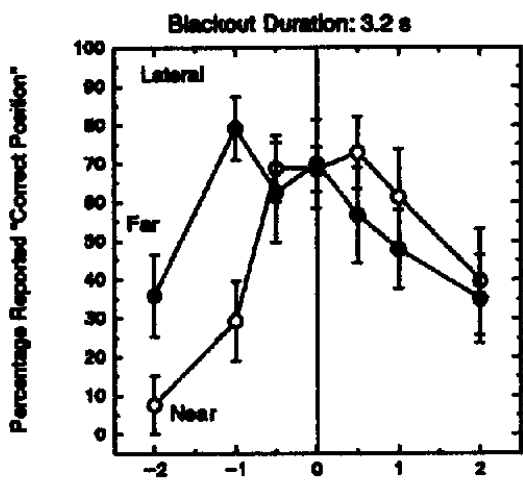

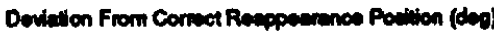

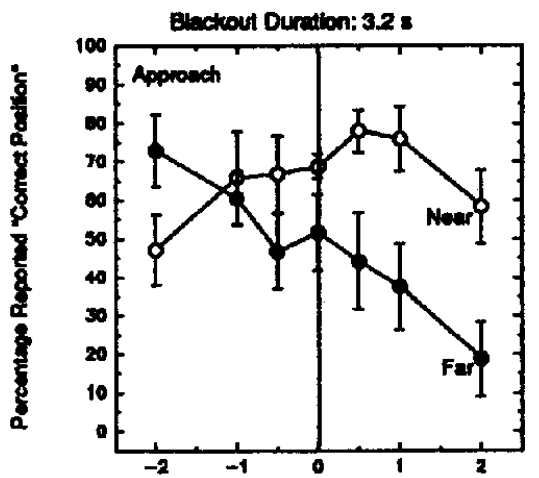

Duvition From Correct Remppestance Potition (deo)

Deviation From Correct Resposarance Poeition (deg)

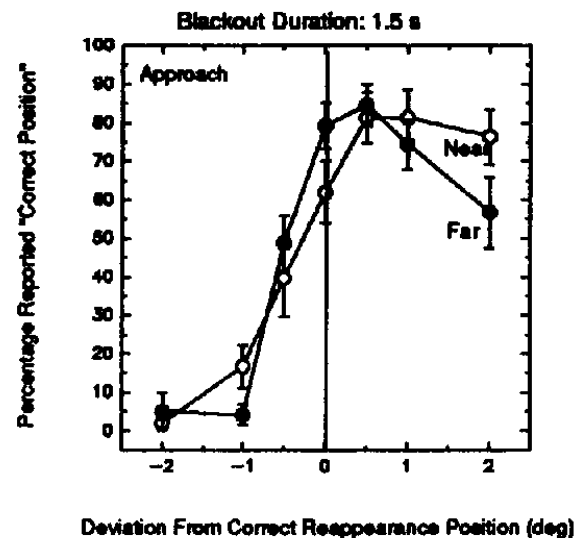

Figure 2. Experiment 1: mean percentage of trials in which observers reported that the object reappeared at the correct position as a function of the deviation between the actual reappearance position and the objectively correct reappearance position for each motion trajectory. Negative and positive values on the horizontal axis indicate undershoots and overshoots, respectively. Error bars indicate $\pm 1 S E M$. 
$.001, R^{2}=56.76 \%$; quadratic, $F(1,46)=22.02, p<.001$, $R^{2}=12.84 \%$; and cubic, $F(1,45)=23.32, p<.001, R^{2}=$ 9.15\%. The mean percentage reported to be correct for overshoots was significantly greater than that for undershoots, $F(1,7)=98.96, p<.0001$. Along with this result, a peak at $0.5^{\circ}$ suggested a bias toward overshoots.

With the 3.2-s blackout duration, the curve appeared to be flatter than that obtained with the 1.5-s blackout duration, indicating that discrimination among the reappearance positions deteriorated. The linear and quadratic components were significant and accounted for comparable amounts of the variance: linear, $F(1,47)=6.66, p<.025, R^{2}=8.58 \%$; quadratic, $F(1,46)=4.80, p<.05, R^{2}=5.72 \%$. The difference between the means of overshoots and undershoots was not significant (but see the following section, Interactive effects of final distance). We distinguished this absence of a response bias from that obtained with lateral motion; in the latter, the response curves were not flat but were fairly symmetrical around the correct reappearance position. The flatter curve obtained with approach motion and a 3.2-s blackout duration was consistent with the deterioration in performance that occurred as TTC increased in the PM task.

However, we expected judgments of approach motion to be biased toward large overshoots. Although the mostly flat response curve indicated poor discrimination among the reappearance positions and thus was generally consistent with the deterioration in performance that occurred as TTC increased in PM tasks, such results may indicate that performance in the IP was not as good as performance in the PM task (see also the section on Weber fractions).

Results of an ANOVA indicated a main effect of reappearance position, $F(6,42)=14.40, p<.0002, M S E=530.28$, $\omega^{2}=17.70 \%$, and its interaction with blackout duration, which accounted for the most variance, $F(6,42)=29.60$, $p<.0001, M S E=346.61, \omega^{2}=24.70 \%$. There were also significant interactions between reappearance position and final distance and between blackout duration and final distance, as well as a three-way interaction among reappearance position, blackout duration, and final distance, $F(6,42)=3.35, p<.0351, M S E=322.23, \omega^{2}=1.88 \%$.

Interactive effects of final distance. The object's final distance was manipulated to be consistent with the design of displays in previous TTC studies (Schiff \& Detwiler, 1979); previous results suggest that distance and velocity are not importantly related to the accuracy of TTC judgments (see Schiff \& Detwiler, 1979; Schiff \& Oldak, 1990; velocity is, of necessity, partly confounded with TTC in this method). In Experiment 1, the interactive effects of final distance, reappearance position, and blackout duration were relatively small. However, such effects deserve comment in light of findings that the accuracy of TTC judgments increased when velocity increased (Schiff, Oldak, \& Shah 1992).

Results indicate a relatively greater bias toward overshoots with near-distance scenes than far-distance scenes (see Figure 2). Specifically, with the 1.5-s blackout duration, the peak of the response curve for lateral motion occurred at the objectively correct position with the far distance and at a $0.5^{\circ}$ overshoot with the near distance (in the latter, the mean for overshoots was significantly greater than that for under- shoots). With approach motion, the mean for overshoots was significantly greater than that for undershoots with both distances, and observers selected the larger overshoots more often with the near distance than with the far distance. With the 3.2-s blackout duration, the peak of the response curves for far-distance scenes indicated a bias toward undershoots with both lateral and approach motion. The peaks of the near-distance scenes indicated a bias toward the $0.5^{\circ}$ overshoot with both trajectories (although the means of overshoots and undershoots were not significantly different). With a 3.2-s blackout duration, the bias toward undershoots suggested by the peaks of the far-distance scenes (although the means of undershoots and overshoots were not signifcantly different for lateral motion) seems inconsistent with the underestimations of TTC with approach motion and the relatively accurate judgments of lateral motion that are typical of PM tasks (Schiff \& Detwiler, 1979; Schiff \& Oldak, 1990).

In summary, in some conditions, the pattern of responses in the IP was consistent with that obtained in previous TTC studies, especially with lateral motion. The pattern of responses obtained with approach motion in the IP seemed less consistent than that obtained with lateral motion. Results of approach motion might have been limited by the small number and range of reappearance positions that we used, which might have reduced the effectiveness of the IP to measure relatively large extrapolation errors, typical of approach motion in the PM task (e.g., see Schiff \& Oldak, 1990).

Control study. In a control study, we measured observers' estimates of TTC with a PM task to determine whether our displays would produce the pattern of results reported in previous studies of TTC. With variations of the scenes in Experiment 1, the object disappeared when it was 0.75, 1.5, 3.2 , or $6 \mathrm{~s}$ from virtual contact with the finish line and did not reappear. ${ }^{4}$ The observers were instructed to press a mouse button when they thought the object would have reached the finish line had it kept moving at the same speed after it disappeared. ${ }^{5}$ Consistent with the pattern of results reported in previous studies, estimated TTC increased as actual TTC increased, and judgment accuracy decreased with larger TTCs and consisted primarily of underestimations. The mean percentage accuracy (judged TTC $* 100$ /actual TTC; Schiff \& Oldak, 1990; Schiff et al., 1992) was significantly greater with lateral motion than with approach motion with the near distance and with the far distance when TTC was $0.75 \mathrm{~s}$. In addition, the percentage accuracy was greater with

\footnotetext{
${ }^{4}$ We used the same apparatus as in Experiment 1. Results of an experiment with modifications of a subset of scenes in the control study suggested that the degree of simulated computer aliasing (see Watt, 1989) or irregularity in an object's optical expansion (which varies with display resolution) does not affect time-to-contact estimates (see also DeLucia, 1991).

${ }^{5}$ All observers reported that they mentally extrapolated or imagined the object's motion when it disappeared. Also, when 8 different observers were instructed explicitly to mentally extrapolate or continue the object's motion in their mind after it disappeared, a comparison of the results with those of the control study indicated no effect of instructions.
} 
Table 3

Experiment 1: Weber Fractions (Expressed as Percentages) and $d^{\prime}$

\begin{tabular}{|c|c|c|c|c|c|c|c|c|}
\hline \multirow[b]{3}{*}{ Final distance } & \multicolumn{4}{|c|}{ Lateral } & \multicolumn{4}{|c|}{ Approach } \\
\hline & \multicolumn{2}{|c|}{ 1.5-s blackout } & \multicolumn{2}{|c|}{ 3.2-s blackout } & \multicolumn{2}{|c|}{ 1.5-s blackout } & \multicolumn{2}{|c|}{ 3.2-s blackout } \\
\hline & $-2^{\circ}$ & $+2^{\circ}$ & $-2^{\circ}$ & $+2^{\circ}$ & $-2^{\circ}$ & $+2^{\circ}$ & $-2^{\circ}$ & $+2^{\circ}$ \\
\hline $\begin{array}{c}\text { Near } \\
d^{\prime} \\
\text { Far } \\
d^{\prime}\end{array}$ & $\begin{array}{r}68.47 \\
2.62 \\
30.94 \\
2.89\end{array}$ & $\begin{array}{r}162.16 \\
1.11 \\
65.92 \\
1.36\end{array}$ & $\begin{array}{r}42.13 \\
2.02 \\
48.30 \\
0.88\end{array}$ & $\begin{array}{r}121.70 \\
0.70 \\
45.32 \\
0.94\end{array}$ & $\begin{array}{r}64.20 \\
2.36 \\
62.60 \\
2.59\end{array}$ & $\begin{array}{r}-344.70 \\
-0.44 \\
246.34 \\
0.66\end{array}$ & $\begin{array}{r}74.47 \\
0.58 \\
-56.62 \\
-0.58\end{array}$ & $\begin{array}{r}144.06 \\
0.30 \\
36.45 \\
0.90\end{array}$ \\
\hline
\end{tabular}

the far final distance (faster velocity) than the near final distance, which is consistent with previous results (Schiff et al., 1992); the means indicated underestimations of TTC in near-distance scenes. The latter findings are consistent with results of Experiment 1, in which near scenes were relatively more biased toward overshoots. Note that in Experiment 1 and in the control study, the different biases in near- and far-distance scenes could be partly associated with a potential methodological artifact of regression toward the mean; observers may select as correct shorter distances and times than average in near-distance scenes and longer distances and times than average in far-distance scenes (J. S. Lappin, personal communication, September 28, 1996).

Weber fractions. Finally, we estimated discriminability with Weber fractions, shown in Tables 3, 4, 5, and 6. ${ }^{6}$ Note that a potential inadequacy of the IP is that observers discriminated among seven reappearance events with only two responses (correct vs. incorrect); when observers responded "incorrect," it was not possible to determine whether they thought the reappearance position was an overshoot or an undershoot (J. S. Lappin, personal communication, January, 17, 1997). Thus, we computed such fractions only for the largest undershoots and overshoots $\left(2^{\circ}\right)$ because in these conditions it was most reasonable to assume that when the observers reported "incorrect" they could discriminate between undershoots and overshoots. In both Experiment 1 and the control study, the Weber fractions for far final distances and lateral motion were typically smaller (i.e., discriminability was better) than those for near distances and approach motion, respectively. However, Weber fractions in the control study were smaller than those in Experiment 1. Also, the Weber fractions indicated particularly poor discrimination in Experiment 1. Such results suggest that the two tasks may not be limited by the same stimulus information and may rely on different underlying processes (J. S. Lappin, personal communication, January $17,1997)$.

\section{Experiment 2}

In Experiment 2, we used a different method to evaluate whether observers would use CME and imagery in judgments of approach motion. We measured observers' judgments about when an approaching object would reach them and varied visual information about the environment between the observer and the display. We reasoned that if observers mentally continue or imagine the object moving through the environment between them and the display after the object disappears, they would use landmarks or contextual cues as references to keep track of the imagined object's position. Thus, performance would deteriorate if such cues were minimized. In other words, we assumed that environmental cues would facilitate visualization, or the tracking of an imagined object that presumably occurs after the object disappears.

\section{Method}

Participants. Sixteen undergraduates from Texas Tech University participated in this experiment.

Displays. Displays consisted of perspective white-on-black drawings of 3-D scenes in which a square object approached the observer while located on a ground plane covered with a grid pattern. The optical parameters of the object are shown in Table 7 and approximated those reported by Schiff and Detwiler (1979, Table 1, small object). The object was stationary for $2 \mathrm{~s}$, approached the observation point for $2 \mathrm{~s}$, and then disappeared when it was $2,4,6,8$, or $10 \mathrm{~s}$ from virtual contact. Two levels of final distance were crossed with TTC, resulting in 10 unique scenes.

Information about the environment between the observer and the display was varied with an aperture. The aperture consisted of a "tunnel" constructed from several file-folder frames connected

'In Experiment 1, we estimated $d$ ' from response frequencies combined across observers in each experimental condition for scenes with a $2^{\circ}$ undershoot and $2^{\circ}$ overshoot. Note that combining frequencies across observers may artificially lower the $d^{\prime}$ values compared with $d^{\prime}$ values computed for each individual and condition and then averaged across observers (J. S. Lappin, personal communication, January 18, 1997). A "hit" was defined as a trial in which the observer reported that the object reappeared in an incorrect position when the position was incorrect; we converted the probabilities of hits and false alarms to $d^{\prime}$ using Elliot's (1964) yes-no tables (when the probability was 0 or 1.00 , we used .01 and .99 , respectively, to estimate $d^{\prime}$; DeLucia \& Novak, 1997; for alternative methods, see MacMillan \& Creelman, 1991). We then divided the deviation from the correct reappearance position $\left(2^{\circ}\right)$ by $d^{\prime}$ in each condition. To estimate a Weber fraction, we then divided this value by either the change in the object's position (in degrees) during the blackout for lateral motion or by the object's optical expansion during the blackout for approach motion (J. S. Lappin, personal communication, September 28, 1996). In the other experiments, we estimated the Weber fraction with the ratio of standard deviation to the mean of the time-tocontact estimates for each observer and condition and then averaged across observers. 
Table 4

Control Study: Weber Fractions (Expressed as Percentages)

\begin{tabular}{|c|c|c|c|c|c|c|c|c|}
\hline \multirow[b]{2}{*}{ Final distance } & \multicolumn{4}{|c|}{ Lateral } & \multicolumn{4}{|c|}{ Approach } \\
\hline & $\begin{array}{c}0.75-\mathrm{s} \\
\text { TTC }\end{array}$ & $\begin{array}{l}1.5-\mathrm{s} \\
\text { TTC }\end{array}$ & $\begin{array}{l}3.2-8 \\
\text { TTC }\end{array}$ & $\begin{array}{l}6.0-\mathrm{s} \\
\text { TTC }\end{array}$ & $\begin{array}{c}0.75-\mathrm{s} \\
\text { TTC }\end{array}$ & $\begin{array}{l}1.5-\mathrm{s} \\
\text { TTC }\end{array}$ & $\begin{array}{l}3.2-\mathrm{s} \\
\text { TTC }\end{array}$ & $\begin{array}{l}6.0-\mathrm{s} \\
\text { TTC }\end{array}$ \\
\hline $\begin{array}{l}\text { Near } \\
\text { Far }\end{array}$ & $\begin{array}{l}26.47 \\
19.87\end{array}$ & $\begin{array}{l}20.78 \\
15.38\end{array}$ & $\begin{array}{l}29.03 \\
20.94\end{array}$ & $\begin{array}{l}14.06 \\
16.46\end{array}$ & $\begin{array}{l}42.92 \\
21.55\end{array}$ & $\begin{array}{l}24.38 \\
21.86\end{array}$ & $\begin{array}{l}25.75 \\
19.75\end{array}$ & $\begin{array}{l}18.72 \\
18.47\end{array}$ \\
\hline
\end{tabular}

Note. $\quad$ TTC $=$ time to contact.

together and covered with black felt. The ends of the tunnel were fitted with cardboard and foam board that were cut such that observers could view only the scene. In addition, a curtain was hung such that observers could not see the length of the tunnel. Half the observers viewed the displays through the aperture in a dark room. This condition provided observers with minimal information about the environment between them and the display. The remaining observers viewed the displays without an aperture in a fully illuminated room, which provided observers with optimal information about the environment. The approach scenes (and thus optical TTC) remained constant as viewing condition varied, and each condition was balanced for gender.

Design and procedure. Observers viewed the displays monocularly (to facilitate comfortable viewing through the aperture) from $2.48 \mathrm{~m}$, and head movements were minimized with a chin rest. The observers were instructed to press a mouse button when they thought the object would have reached them had it kept coming at the same speed after it disappeared. Judgments of TTC were measured by the time between the object's disappearance and the observer's response. All scenes were presented four times in a random order. Ten practice trials were provided but were not analyzed.

\section{Results and Discussion}

Results are summarized in Figure 3. A $2 \times 2 \times 5$ ANOVA (Viewing Condition $\times$ Final Distance $\times$ TTC) with repeated measures on the last two variables indicated a significant interaction between viewing condition and final distance, $F(1,14)=5.67, M S E=1.43, p<.032$, which accounted for less than $1 \%$ of the variance. Analyses of simple main effects indicated that, with the near final distance, TTC estimates were greater when displays were

Table 5

Experiment 2 (Approach): Weber Fractions

(Expressed as Percentages)

\begin{tabular}{lccccc}
\hline \multirow{6}{*}{$\begin{array}{c}\text { Final } \\
\text { distance }\end{array}$} & 2 & 4 & 6 & 8 & 10 \\
\cline { 2 - 6 } Aperture \\
Near & 44.06 & 29.44 & 29.82 & 33.51 & 33.06 \\
Far & 27.38 & 30.27 & 26.61 & 26.93 & 28.32 \\
\hline \multicolumn{5}{c}{ Nonaperture } \\
Near & 27.46 & 25.16 & 19.12 & 28.12 & 26.49 \\
Far & 17.44 & 25.70 & 20.75 & 35.77 & 41.33 \\
\hline
\end{tabular}

viewed without an aperture, $F(1,15)=4.59, p<.049$. The same pattern of means occurred with the far final distance, but the differences were not statistically significant.

Although the main effect of viewing condition was not significant, it accounted for a substantial $8.58 \%$ of the variance. Moreover, the results suggest that the relationship between estimated and actual TTC depends on the viewing condition (see Figure 3). The line that best fit the relationship was approximated with statistical regression techniques. In the nonaperture condition, the slopes were .69 and .62 for near and far distances, respectively, which approximated those reported in earlier studies (.58 in McLeod \& Ross, 1983 ; 61 in Schiff \& Detwiler, 1979). With aperture viewing, the corresponding slopes were .35 and .43 . Thus, slopes decreased or performance deteriorated when visual information about the environment was minimized. Furthermore, the Weber fractions were typically larger in the aperture condition (see Table 5). Results are consistent with our hypothesis that observers imagined the object moving through the environment between them and the display. In the near and far conditions, the slopes for the aperture condition were $49 \%$ and $31 \%$ smaller, respectively, than the slopes for the nonaperture conditions.

The evidence for CME in Experiment 2 is only suggestive. Moreover, even though the visual display (i.e., optical TTC) did not vary when the aperture was added, we cannot be certain that for some reason the aperture did not affect the perception of optical TTC when the approaching object was visible. However, if information about the environment between the observer and the display affects TTC judgments in at least some conditions, the implication is that the CME that presumably underlies such effects may contribute to TTC judgments in PM tasks.

Table 6

Experiment 3 (Lateral): Weber Fractions (Expressed as Percentages)

\begin{tabular}{|c|c|c|c|c|c|c|}
\hline \multirow[b]{2}{*}{$\begin{array}{c}\text { Final } \\
\text { distance }\end{array}$} & \multicolumn{2}{|c|}{$\begin{array}{c}\text { Control } \\
\text { task }\end{array}$} & \multicolumn{2}{|c|}{$\begin{array}{l}\text { Auditory } \\
\text { condition }\end{array}$} & \multicolumn{2}{|c|}{$\begin{array}{l}\text { Visual } \\
\text { condition }\end{array}$} \\
\hline & $\begin{array}{l}3.2-\mathrm{s} \\
\text { TTC }\end{array}$ & $\stackrel{6-s}{\text { TTC }}$ & $\begin{array}{l}3.2-\mathrm{s} \\
\text { TTC }\end{array}$ & $\begin{array}{c}\text { 6-s } \\
\text { TTC }\end{array}$ & $\begin{array}{l}3.2-\mathrm{s} \\
\text { TTC }\end{array}$ & $\stackrel{\text { 6-s }}{\text { TTC }}$ \\
\hline $\begin{array}{l}\text { Near } \\
\text { Far }\end{array}$ & $\begin{array}{l}20.39 \\
18.71\end{array}$ & $\begin{array}{l}14.45 \\
12.98\end{array}$ & $\begin{array}{l}13.97 \\
19.10\end{array}$ & $\begin{array}{r}13.59 \\
8.77\end{array}$ & $\begin{array}{l}13.51 \\
15.84\end{array}$ & $\begin{array}{r}12.17 \\
9.37\end{array}$ \\
\hline
\end{tabular}

Note. TTC $=$ time to contact. 
Table 7

Experiment 2: Degrees of Visual Angle Subtended by the Object's Height

\begin{tabular}{clll}
\hline $\begin{array}{c}\text { TTC } \\
\text { (in seconds) }\end{array}$ & $\begin{array}{c}\text { Final } \\
\text { distance }\end{array}$ & $\begin{array}{c}\text { First } \\
\text { frame }\end{array}$ & $\begin{array}{c}\text { Last } \\
\text { frame }\end{array}$ \\
\hline 2 & Near & 0.859 & 1.727 \\
2 & Far & 0.430 & 0.866 \\
4 & Near & 1.146 & 1.723 \\
4 & Far & 0.573 & 0.862 \\
6 & Near & 1.292 & 1.727 \\
6 & Far & 0.646 & 0.864 \\
8 & Near & 1.375 & 1.721 \\
8 & Far & 0.688 & 0.862 \\
10 & Near & 1.432 & 1.719 \\
10 & Far & 0.716 & 0.860 \\
\hline
\end{tabular}

Note. $\quad$ TTC $=$ time to contact.

\section{Application of Selective Interference to a PM Task}

In Experiment 1, we compared the pattern of results previously obtained with the PM task, which could involve CME or a clocking process that counts down TTC, with the results of an IP task in which, we argue, the latter clocking process is not viable. We assumed that the similarity in results between the IP and PM tasks suggests that the PM tasks involves CME. However, such evidence for CME is indirect. In Experiment 2, we attempted to affect the use of CME and imagery in judgments of approach more directly, but the results were only suggestive.

In Experiment 3, we considered selective interference or concurrent processing (e.g., Brooks, 1967, 1968; Fortin \& Breton, 1995; Fortin, Rousseau, Bourque, \& Kirouac, 1993; Segal \& Fusella, 1970; Wickens, 1992) to evaluate the processes that underlie the PM task. In such a method, observers perform an interference task while they perform a PM task (for a related method, see Bardy \& Laurent, 1991). The additional task is designed to interfere with either CME or a clocking process that relies on optical TTC information. It is assumed that performance will deteriorate only if the

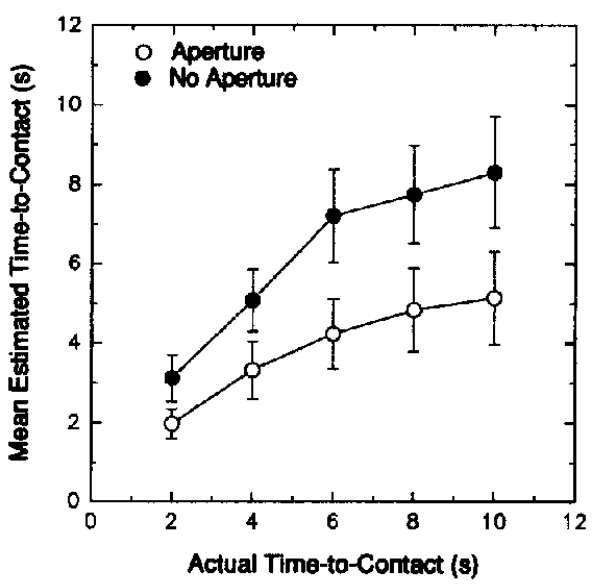

Figure 3. Experiment 2: mean time-to-contact (TTC) estimates as a function of actual TTC. Error bars indicate \pm 1 SEM.
PM task and interference task demand common resources (e.g., Wickens, 1992). Thus, by carefully designing the interference task (and making certain assumptions), one potentially can elucidate the nature of the processes that underlie the PM task.

The use of selective interference to determine whether observers use CME or a clocking process that relies on optical TTC in the PM task poses many issues. For example, the specific nature of the clocking process that presumably underlies the PM task must be defined before the interference task can be designed. A specific model of a clocking process in PM tasks (Tresilian, 1995; see also Lyon \& Waag, 1995) was not discussed.

However, it is reasonable to assume that the timing mechanism or clock that potentially underlies PM tasks is the same timing mechanism discussed in studies of time perception (e.g., Block, 1990; Woodrow, 1951). Indeed, previous results suggest that both time perception and motor action use the same or similar timing mechanisms (Treisman, Faulkner, \& Naish, 1992). Nevertheless, an assumption that the same clocking process is used in PM tasks and time perception does not solve the problem of designing an interference task because there are several models of time estimation (for a review, see Block, 1990). For example, it has been suggested that time estimation involves attentional resources (e.g., Brown \& West, 1990), short-term or working memory (e.g., Fortin \& Breton, 1995; Fortin et al., 1993), and a temporal oscillator (Treisman, Faulkner, Naish, \& Brogan, 1990). Furthermore, results of time-estimation studies are variable and depend on the method (see Zakay, 1990).

Moreover, even if a particular clock is defined, there are issues that must be addressed that are specific to the aim of distinguishing between CME and a clocking process that relies on optical TTC in the PM task. For example, CME likely involves some type of temporal process. Thus, a temporal judgment may interfere with both CME and a clocking process that counts down TTC. Similarly, nontemporal tasks can interfere with time estimation (e.g., Fortin et al., 1993). Therefore, one cannot assume that a nontemporal judgment would interfere with CME but not with a clocking process. In Experiment 3, we addressed these issues and applied selective interference to a PM task.

\section{Experiment 3}

While observers performed a PM task, they also judged the relative temporal duration of two stimuli that were presented either visually or aurally. If the PM task involves only a clocking process that counts down TTC, there should be no difference in performance between the visual and auditory conditions. Alternatively, if the PM task involves CME, there should be a greater performance decrement in the visual task condition than in the auditory task condition. In other words, a PM task and a concurrent relative duration task should result in degraded performance compared with a PM task alone, and this decrement should be relatively greater in the visual task condition. This would be generally 
consistent with previous studies of selective interference (e.g., Brooks, 1967; Segal \& Fusella, 1970).

\section{Method}

Participants. Eight undergraduates from Texas Tech University participated in this experiment. ${ }^{7}$

Displays. The displays consisted of black-on-white perspective drawings of a subset of the lateral motion scenes from the control study (the TTC was $3.2 \mathrm{~s}$ and $6 \mathrm{~s}$ ). On interference trials, additional stimuli were presented $641 \mathrm{~ms}$ after the moving object disappeared. In the visual interference (VI) condition, the stimuli were located about $9.72^{\circ}$ to the left and $6.87^{\circ}$ above the observation point. Two parallel lines with a horizontal dimension of $0.78^{\circ}$ were presented successively, separated by $214 \mathrm{~ms}$. Their vertical dimension in virtual space was such that each line was 1 pixel in thickness (approximately 2.9 arcmin). One line was located below a 1-pixel reference dot (the "lower line"), and the other was located above the dot (the "higher line"). The dot was centered horizontally and vertically between the lines and was $0.17^{\circ}$ from each line $(\Xi)$. On half the trials, one line was $299 \mathrm{~ms}$ in temporal duration and the other was $470 \mathrm{~ms}$; in this case the lower line was presented before the higher line. On the remaining trials, one line was $384 \mathrm{~ms}$ in duration and the other was $769 \mathrm{~ms}$; in this case the higher line was shown before the lower line. In the auditory interference (AI) condition, a low-pitched tone and a high-pitched tone (approximately 400 and $800 \mathrm{~Hz}$, respectively) were presented successively, separated by $214 \mathrm{~ms}$. The durations of the tones approximated the durations of the horizontal lines. ${ }^{8}$ The relative durations of visual and auditory stimuli were above discrimination thresholds for comparative time estimation, as reported previously (Woodrow, 1951). The stimulus that appeared first was not always lower (in pitch or spatial position) or longer in duration. Finally, at least $1.15 \mathrm{~s}$ elapsed after the second stimulus was presented and before TTC occurred.

Design of the interference tasks. In designing the VI and AI tasks, we assumed that CME, which potentially underlies PM tasks, relies on visuospatial and temporal processes (as described earlier), whereas a clocking process that is used to count down TTC after the object disappears (Tresilian, 1995) involves only a temporal process. We also assumed that the temporal processes are of the same class of mechanisms implicated in time estimation by Fortin and Breton (1995); their results suggest that visuospatial processing in working memory can interfere with concurrent time estimation.

We selected relative duration as an interference task for several reasons. First, it involves a temporal judgment and thus should interfere with temporal processes used in the PM task. Second, we assumed that the relative judgment involves short-term or working memory and would interfere with the temporal processes implicated by Fortin (Fortin \& Breton, 1995; Fortin et al., 1993). However, our Experiment 3 was not designed to distinguish the temporal process that is the basis of a clock that counts down TTC from the process that is potentially used in CME (and which does not necessarily rely on optical TTC). Third, compared with a temporal production or reproduction task, relative duration seems less susceptible to counting strategies (see Lyon \& Waag, 1995; Zakay, 1990) and to delays that can occur when two responses are made serially and close in time (see Wickens, 1992).

Finally, we did not compare the results of a temporal interference task and a nontemporal spatial interference task because it is likely that a nontemporal spatial task would interfere with a temporal process used in the PM task (see Fortin et al., 1993). Moreover, it would be difficult to equate the complexity of such different tasks.
We did not want the two tasks to affect performance differently simply because of differences in task complexity (see Zakay, 1990).

Design and procedure. All observers participated in three conditions. In the control condition, the observers were instructed to press the $F$ key on a computer keyboard when they thought the object would have reached the finish line had it kept moving at the same speed after it disappeared. In the VI condition, after observers completed the PM task, they reported verbally whether the lower line or the higher line was longer in duration. In the AI condition, after observers completed the PM task, they reported verbally whether the low-pitched tone or the high-pitched tone was longer in duration. We instructed observers to make the PM response first to maximize the interference between the $\mathrm{PM}$ and relative duration tasks. Similarly, in the AI and VI conditions, the observers were informed that the PM task and the relative duration task were equal in importance, and they were instructed to perform both tasks as best as they could. We provided feedback on the relative duration task to encourage observers to perform the task.

Sixteen trials were presented in a random order in each of the control, AI, and VI conditions; there were four replications for each combination of TTC and final distance. The order of conditions was either control, VI, AI; control, AI, VI; VI, AI, control; or AI, VI, control. It was counterbalanced across observers and balanced for gender. We measured judgments of TTC by the time between the object's disappearance and the observer's response. We also measured the percentage accuracy of relative duration judgments. We deleted trials in which observers responded before the object disappeared and in which observers gave the verbal response before the $F$-key response. Eight practice trials were provided before each block of trials but were not analyzed.

\section{Results and Discussion}

Estimates of TTC. Results are summarized in Figure 4, and Weber fractions are shown in Table 6. Trials in which the observers responded incorrectly on the AI and VI tasks were deleted. Results of a $2 \times 2 \times 3$ repeated measures ANOVA (TTC $\times$ Final Distance $\times$ Interference Condition) indicated that differences in mean TTC estimates among the control, $\mathrm{AI}$, and VI conditions were not significant. The means were $4.04,4.20$, and $3.92 \mathrm{~s}$, respectively. There was a main effect

\footnotetext{
${ }^{7}$ One observer was replaced because of poor performance, which resulted in missing data in the analysis of time-to-contact estimates.

${ }^{8}$ Our description of the tone parameters was accurate to within the limits of the computer speaker and clock. Also, we displaced the visual interference (VI) stimulus from the object's path to make it difficult to follow the hidden motion while judging the stimulus with peripheral vision (which may minimize effort on the VI task). This was not germane to the auditory interference task; we considered this potential difference between the two tasks to be negligible because the VI stimulus appeared $641 \mathrm{~ms}$ after the object disappeared, presumably permitting observers to shift fixation before its onset. Also, a pilot study indicated that performance on a prediction motion task (and a concurrent visual judgment of whether a line was continuous or interrupted) obtained when the interference stimulus was in the object's path was comparable to that obtained when the stimulus was displaced from the path. Furthermore, it was reported that position prediction was equivalent when eye tracking was encouraged and when it was prevented (Peterken, Brown, \& Bowman, 1991). Finally, we could not rule out the role of eye movements or covert attentional shifts even if the VI stimulus was placed in the object's path.
} 

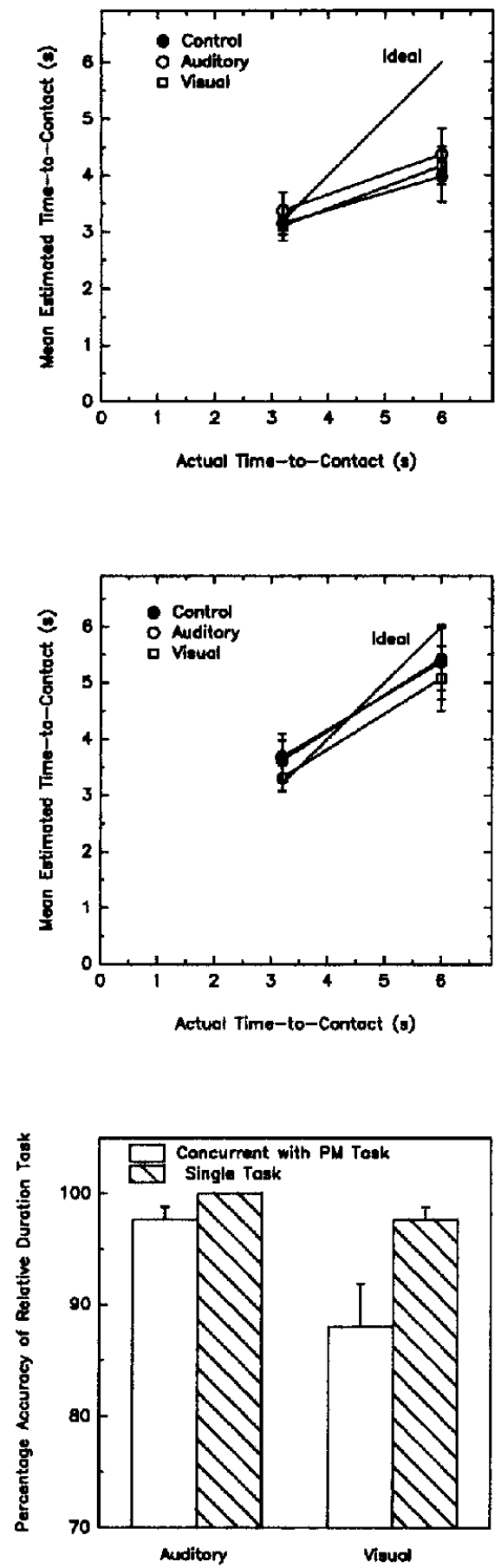

Figure 4. Experiment 3: mean time-to-contact (TTC) estimates as a function of actual TTC for near final distance (top panel) and far final distance (middle panel) and percentage accuracy in the relative duration task for concurrent and single-task conditions (bottom panel). Error bars indicate \pm 1 SEM. $\mathrm{PM}=$ prediction motion.

of TTC, $F(1,7)=37.03, p<.0005, M S E=1.20, \omega^{2}=$ 21.94\%; final distance, $F(1,7)=26.61, p<.0013, M S E=$ $0.47, \omega^{2}=6.11 \%$; and their interaction, $F(1,7)=32.71$, $p<.0007, M S E=0.11, \omega^{2}=1.85 \%$.
Relative duration judgments. Results of the relative duration judgments are summarized in Figure 4. The absence of an effect of the interference tasks on estimated TTC may indicate that neither CME nor a clocking process that counts down TTC is involved in the PM task. However, interference also could be reflected as a decrement in the relative duration judgment, especially if observers considered the PM task as their primary or more difficult judgment and performed it at the expense of the relative duration judgment (see Fortin et al., 1993; Gopher \& Donchin, 1986; Wickens, 1992).

Indeed, results of a two-tailed $t$ test indicated that mean accuracy (averaged across all scenes) was significantly lower in the VI task than in the AI task, $t(7)=2.90, p<.05$ (the respective means were $97.7 \%$ and $88.0 \%$ ). Furthermore, we instructed observers to rate the difficulty of the PM task in the AI and VI conditions separately; we used a 7-point scale in which higher values indicated greater difficulty. The mean rating in the VI condition was significantly greater than in the AI condition, $t(7)=6.12, p<.001$ (the respective means were 4.75 and 2.75 ).

Although we attempted to design the AI and VI conditions to be comparable in difficulty, it is possible that the VI task was more difficult than the AI task regardless of whether the PM task was performed concurrently. Thus, we instructed 8 different observers to perform the relative duration task without performing the PM task. They viewed the same stimuli as in Experiment 3 but were instructed to press the $F$ key when they thought they knew which stimulus was longer in duration; they then reported their answer verbally. Results indicated that the difference in mean accuracy between the AI and VI conditions was not significant (the means were $100 \%$ and $97.7 \%$ ); the difference in mean difficulty ratings also was not significant (the respective means were 1.6 and 2.1). An expected difference between the two groups (who did the tasks either alone or concurrently with the PM task) in the differential accuracy between $\mathrm{AI}$ and VI tasks was not significant.

More important, the mean accuracy that was obtained when the PM task and VI task were done concurrently was significantly lower than that obtained when the VI task was performed alone, $t(14)=2.41, p<.05$. The analogous comparison was not significant in the AI condition. Results suggest that differences in accuracy between the $\mathrm{AI}$ and VI tasks in Experiment 3 were attributable to the concurrent PM task rather than to differences in task difficulty.

Results are consistent with the notion that PM tasks involve CME rather than solely a clocking process that counts down TTC. Our results also are consistent with those of Lyon and Waag (1995), who reported that a distractor can affect extrapolation accuracy under certain conditions and suggested that some kind of tracking mechanism was at least a component of visual extrapolation performance in their study.

\section{Summary and General Discussion}

We hypothesized that observers cognitively extrapolate motion to aid their judgments in the PM task and that such 
CME is accompanied by imagery. In Experiment 1, we proposed that if observers use CME in the PM task, the pattern of response errors obtained in the IP task would be similar to that obtained in previous studies of PM tasks. In some conditions, the pattern of responses in the IP was consistent with that obtained in previous PM tasks, especially with lateral motion. This suggests that a cognitive clock based on optical TTC is not necessary to obtain a pattern of extrapolation errors that characterizes performance in the PM task. However, the pattern of results with approach scenes seems less consistent than that of lateral scenes; the results of far-distance scenes and a 3.2-s blackout duration (with both trajectories) seem inconsistent with results of PM tasks; and the IP provides only indirect or inferential evidence of CME in the PM task. Furthermore, Weber fractions were higher in the IP task than in the PM task and suggest particularly poor discriminability in the IP; this may indicate that the IP and PM tasks are not limited by the same stimulus information, and rely on different underlying processes, or that our IP method may not provide an adequate measure of discriminability.

Moreover, we acknowledge that the classification of processes that we consider to potentially underlie PM tasks can be characterized as one in which such processes are either spatial or temporal and that our method is limited in distinguishing such processes (J. R. Tresilian, personal communication, March 21, 1996). That is, observers may compute TTC from an internal spatial representation of the object's visible motion; this can be considered a spatial extrapolation process with time only as an implicit parameter. Alternatively, observers may use a simple temporal waiting process with an internal clock as described earlier; this can be considered an explicitly temporal process that does not involve a spatial representation. Therefore, if the pattern of performance in the IP task (which involves a spatial representation) is similar to the pattern of performance in the PM task (which could involve either a spatial or explicitly temporal process), the implication is that the process that underlies the PM task is spatial rather than temporal. However, such an approach assumes that similarities in performance between the IP and PM tasks occur because the extrapolation processes share the same dimension (spatial or temporal). We cannot rule out the possibility that the similarities are due to general properties common to all such processes regardless of whether they are spatial or temporal.

In Experiment 2, performance deteriorated when information about the environment was minimized with an aperture. This is consistent with the notion that in the PM task, observers imagine that the object moves through the environment between them and the display after the object disappears. However, the results were only suggestive.

Finally, the results of Experiment 3 also are consistent with the notion that PM tasks involve CME rather than solely a clocking process that counts down TTC. Specifically, the accuracy of relative duration judgments of visual (but not auditory) stimuli decreased significantly when a PM task was performed concurrently. This suggests that performance in the PM task and the VI task (but not the AI task) demand common resources. However, we cannot rule out the possibility that our interference tasks changed the strategy or process involved in the PM task (see also Gopher \& Donchin, 1986; Wickens, 1992).

Our results have several implications: First, they suggest that PM tasks do not represent a pure measure of an observer's ability to use optical TTC. In at least some conditions, observers may rely on cognitive operations, particularly on CME with imagery. Therefore, the pattern of results from PM tasks that was reported in previous studies may reflect such CME rather than, or in addition to, the use of optical TTC. It is important to reevaluate previous conclusions made on the basis of PM tasks about the efficacy with which observers use optical TTC information.

Second, it has been argued that whether observers use cognitive extrapolation in PM tasks depends on the theoretical perspective that one espouses (Schiff \& Oldak, 1990). The PM task alone does not facilitate empirical evaluation of whether observers use CME. We began to address this issue with three different methods. It seems particularly useful to extend Experiment 3 with different interference tasks. For example, a mental rotation task (Cooper \& Shepard, 1973) should interfere with visuospatial processes (e.g., CME) that potentially occur in the PM task and thereby affect concurrent TTC judgments (Liddell, 1998). This is a reasonable proposal especially because previous results suggest that both time perception and motor action use the same or similar timing mechanisms (Treisman et al., 1992) and that visuospatial processing in working memory can interfere with concurrent time estimation (Fortin \& Breton, 1995).

Third, it has been suggested that TTC judgments of approach motion are less accurate than those of lateral motion because optical changes are nonlinear in the former and approximately linear in the latter and that observers may use different visual information for each trajectory (Schiff \& Oldak, 1990). Similarly, observers may have used a different strategy (CME or a clock) for each trajectory in our experiments (M. K. Kaiser, personal communication, March $21,1996)$. Our study was not designed to address this issue. ${ }^{9}$ We note that judgments of approach motion were typically less accurate than judgments of lateral motion in the IP (in which TTC is not relevant). One implication is that the effects of trajectory in PM tasks may reflect differences in CME of lateral and approach motion rather than, or in addition to, differences in the efficacy with which observers extract TTC from different optic flow patterns.

In conclusion, our results are mostly consistent with the notion that PM tasks involve CME rather than solely a

\footnotetext{
${ }^{9}$ Previous prediction motion studies did not include object rotation, which results in linear optical changes, similar to lateral motion. We replicated the control study using a rotating cube; observers predicted when a landmark on the cube would "reach" or become parallel with an adjacent "finish line." The effects of time to contact (TTC) were similar to those in the control study. Also, the percentage accuracy was greater with rotation scenes than approach scenes (from the control study) when TTC was $6 \mathrm{~s}(78 \%$ vs. $49 \%$ ) or final distance was near ( $95 \%$ vs. $68 \%$ ), suggesting that linearity of optical changes can affect TTC judgments.
} 
clocking process that counts down TTC. However, further study is needed to elucidate the nature of the processes that underlie PM tasks. Although we have interpreted our results in terms of underlying cognitive processes, it is possible that our results reflect underlying visual processes (e.g., the results of Experiments 2 and 3 may indicate that TTC judgments are influenced by visual information about space and time, respectively; J. S. Lappin, personal communication, September 28, 1996). It is important to determine the relative contributions of optical TTC and cognitive operations in TTC judgments under various conditions (see also Tresilian, 1995), and to evaluate further the processes that underlie PM tasks, in order to develop a more complete model of TTC judgments. The methods described here warrant further development toward this aim.

\section{References}

Bardy, B. G., \& Laurent, M. (1991). Visual cues and attention demand in locomotor positioning. Perceptual and Motor Skills, 72, 915-926.

Block, R. A. (1990). Cognitive models of psychological time. Hillsdale, NJ: Erlbaum.

Bootsma, R. J., \& Oudejans, R. R. D. (1993). Visual information about time to collision between two objects. Journal of Experimental Psychology: Human Perception and Performance, 19, 1041-1052.

Brooks, L. R. (1967). The suppression of visualization by reading. Quarterly Journal of Experimental Psychology, 19, 289-299.

Brooks, L. R. (1968). Spatial and verbal components of the act of recall. Canadian Journal of Psychology, 22, 349-368.

Brown, S. W., \& West, A. N. (1990). Multiple timing and the allocation of attention. Acta Psychologica, 75, 103-121.

Cooper, L. A. (1989). Mental models of the structure of visual objects. In B. E. Shepp \& S. Ballesteros (Eds.), Object perception: Structure and process (pp. 91-119). Hillsdale, NJ: Erlbaum.

Cooper, L. A., Gibson, B., Mowafy, L., \& Tataryn, D. J. (1987, November). Mental extrapolation of perceptually-driven spatial transformations. Paper presented at the 28th Annual Meeting of the Psychonomic Society, Seattle, WA.

Cooper, L. A., \& Shepard, R. N. (1973). Chronometric studies of the rotation of mental images. In W. G. Chase (Ed.), Visual information processing (pp. 75-176). New York: Academic Press.

DeLucia, P. R. (1991). Pictorial and motion-based information for depth perception. Journal of Experimental Psychology: Human Penception and Performance, 17, 738-748.

Delucia, P. R. (1995). Effects of pictorial relative size and ground-intercept information on judgments about potential collision in perspective displays. Human Factors, 37, 528-538.

DeLucia, P. R., \& Novak, J. (1997). Judgments of relative time-to-contact of more than two approaching objects: Toward a method. Perception \& Psychophysics, 59, 913-928.

DeLucia, P. R., \& Warren, R. (1994). Pictorial and motion-based depth information during active control of self-motion: Sizearrival effects on collision avoidance. Journal of Experimental Psychology: Human Perception and Performance, 20, 783-798.

Elliot, P. B. (1964). Appendix 1. In J. A. Swets (Ed.), Signal detection and recognition by human observers (pp. 651-683). New York: Wiley.

Fortin, C., \& Breton, R. (1995). Temporal interval production and processing in working memory. Perception \& Psychophysics, $57,203-215$.
Fortin, C., Rousseau, R., Bourque, P., \& Kirouac, E. (1993). Time estimation and concurrent nontemporal processing: Specific interference from short-term-menory demands. Perception \& Psychophysics, 53, 536-548.

Gibson, J. J. (1979). The ecological approach to visual perception. Boston: Houghton Mifflin.

Gopher, D., \& Donchin, E. (1986). Workload: An examination of the concept. In K. Boff, J. Thomas, \& L. Kaufman (Eds.), Handbook of perception and human performance (pp. 41-1 to 41-49). New York: Wiley.

Jagacinski, R., Johnson, W., \& Miller, R. (1983). Quantifying the cognitive trajectories of extrapolated movements. Journal of Experimental Psychology: Human Perception and Performance, 9, 43-57.

Kaiser, M. K., \& Mowafy, L. (1993). Optical specification of time-to-passage: Observers' sensitivity to global tau. Journal of Experimental Psychology: Human Perception and Performance, $19,1028-1040$.

Larish, J. F., \& Andersen, G. J. (1995). Active control in interrupted dynamic spatial orientation: The detection of orientation change. Penception \& Psychophysics, 57, 533-545.

Lee, D. N. (1974). Visual information during locomotion. In R. B. McLeod \& H. L. Pick, Jr. (Eds.), Penception: Essays in honor of James J. Gibson (pp. 250-267). Ithaca, NY: Cornell University Press.

Liddell, G. W. (1998). Interfering and updating cognitive representations used in judgments of absolute time-to-contact in a prediction motion task. Dissertation Abstracts International, 58(10), 5678B. (University Microfilms No. AAT 9812032)

Lyon, D. R., \& Waag, W. L. (1995). Time course of visual extrapolation accuracy. Acta Psychologica, 89, 239-260.

Macmillan, N. A., \& Creelman, C. D. (1991). Detection theory: A user's guide. New York: Cambridge University Press.

McLeod, R. W., \& Ross, H. E. (1983). Optic-flow and cognitive factors in time-to-collision estimates. Perception, 12, 417-423.

Novak, J. B. (1998). Judgments of absolute time-to-contact in multiple object displays: Evaluating the role of cognitive processes in arrival-time judgments. Dissertation Abstracts Intermational, 58(10), 5679B. (University Microfilms No. AAT 9812047)

Peterken, C., Brown, B., \& Bowman, K. (1991). Predicting the future position of a moving target. Perception, 20, 5-16.

Rosenbaum, D. A. (1975). Perception and extrapolation of velocity and acceleration. Journal of Experimental Psychology: Human Perception and Performance, 1, 395-403.

Schiff, W., \& Detwiler, M. L. (1979). Information used in judging impending collision. Perception, 8, 647-656.

Schiff, W., \& Oldak, R. (1990). Accuracy of judging time to arrival: Effects of modality, trajectory, and gender. Journal of Experimental Psychology: Human Perception and Peformance, 16, 303-316.

Schiff, W., Oldak, R., \& Shah, V. (1992). Aging person's estimates of vehicular motion. Psychology and Aging, 7, 518-525.

Segal, S. J., \& Fusella, V. (1970). Influence of imaged pictures and sounds on detection of visual and auditory signals. Journal of Experimental Psychology, 83, 458-464.

Todd, J. T. (1981). Visual information about moving objects. Journal of Experimental Psychology: Human Perception and Performance, 7, 795-810.

Treisman, M., Faulkner, A., \& Naish, P. L. N. (1992). On the relation between time perception and the timing of motor action: Evidence for a temporal oscillator controlling the timing of movement. Quarterly Journal of Experimental Psychology Section A: Human Experimental Psychology, 45A, 235-263.

Treisman, M., Faulkner, A., Naish, P. L. N., \& Brogan, D. (1990). The internal clock: Evidence for a temporal oscillator underlying 
time perception with some estimates of its characteristic frequency. Perception, 19, 705-743.

Tresilian, J. R. (1990). Perceptual information for the timing of interceptive action. Perception, 19, 223-239.

Tresilian, J. R. (1991). Empirical and theoretical issues in the perception of time to contact. Joumal of Experimental Psychology: Human Perception and Performance, I7, 865-876.

Tresilian, J. R. (1995). Perceptual and cognitive processes in time-to-contact estimation: Analysis of prediction-motion and relative judgment tasks. Perception \& Psychophysics, 57, 231245.

Watt, A. (1989). Fundamentals of three-dimensional computer graphics. Reading, MA: Addison-Wesley.
Wickens, C. D. (1992). Engineering psychology and human performance (2nd ed.). New York: HarperCollins.

Woodrow, H. (1951). Time perception. In S. S. Stevens (Ed.), Handbook of experimental psychology (pp. 1225-1236). New York: Wiley.

Zakay, D. (1990). The evasive art of subjective time measurement: Some methodological dilemmas. In R. A. Block (Ed.), Cognitive models of psychological time (pp. 59-84). Hillsdale, NJ: Ertbaum.

Received May 2, 1995

Revision received March 3, 1997

Accepted April 8, 1997 